\title{
Gender characteristics of teenage aggression on social networks
}

\author{
Angelica Luchinkina ${ }^{1, *}$ and Vladimir Senchenko ${ }^{1}$ \\ ${ }^{1}$ Crimean Engineering and Pedagogical University, Simferopol, 295015, Russia
}

\begin{abstract}
The article discusses the problems of gender differences in the teenage aggressive behavior on social networks. The article provides a theoretical overview of approaches to the study of the problem of gender differences in aggressive behavior of adolescents, including in the Internet space: psychophysiological, psychosocial, and socio-psychological. The study results showed that the process of aggressive interaction between adolescents on social networks was implemented according to the strategies of psychological defense chosen by adolescents, the level of hostility and self-presentation of an individual through asocial behavior. The authors note that the transition to the virtual space gives adolescents the opportunity to experiment with their own gender, and the number of adolescents with a distinction of biological sex and gender role increases. The study revealed differences in the aggressive behavior between all groups of respondents, depending on their choice of gender role. All results are confirmed using the methods of mathematical statistics.
\end{abstract}

\section{Introduction}

\subsection{Relevance}

The current development stage of society, the general digitalization of the population and the globalization of economic space change the modern way of life not only for adults, but also for children. Adolescents, whose growing up takes place more often in a virtual environment of social media than in the previously familiar social institutions - family and school - are especially vulnerable to the new reality. Teenagers with little social experience are immersed in the virtual space with all the passion inherent in this phase, which can lead to a violation of the assessment of communication situation, wrong perception of reality and misconceptions about self-expression. Experimenting with one's virtual identity involves more than choosing an avatar or status.

Acceptance of a new sociosexual orientation, a new model of behavior appropriate to a new gender, expands the adolescent's social experience, but can lead to deviations of personality and behavior. Every day, thousands of adolescents are bullied and trolled on social networks, while the personality of a bully or a 'troll' remains outside the scope of

\footnotetext{
* Corresponding author: aluch@yandex.ru
} 
psychologist's corrective work, and gender differences in aggressive behavior on social networks are out of the frame of the study.

The scientific problem of the research is to identify gender differences in aggressive behavior of adolescents on social networks, to create a system of preventive work of a psychologist with victims of aggressive behavior and bullies in the Internet space.

According to E. Maccoby and S. Jacklin, aggression is the only social behavior characterized by quite obvious gender differences.

\subsection{Statement of the primary material}

There are several approaches to the question of gender differences in the aggressive behavior of adolescents: psychophysiological, psychosocial, and socio-psychological.

The representatives of the psychophysiological approach focus on the biological properties of the organism and psychophysiological characteristics (Zharnovetskaya 2006; Zhuikova 2014; Ilyin 2014; Isaeva 2008; Kalinin and Urivskaya 2017; Kovalev 1996). Therefore, P.A. Kovalev (1996) defines the aggressiveness of a teenager as a personality trait, pointing out that it reveals itself in emotional state and self-esteem. The scientist emphasizes that girls' sensitivity during puberty is higher than boys' one.

A.B. Kryuchkova (2010) described gender differences in the development of the emotional, productive and regulatory components of the aggressiveness of adolescents.

T.V. Nechepurenko (2009) highlighted gender differences in the manifestations of aggressiveness among adolescents. According to his research, girls are characterized by autoaggression due to affective-aggressive behavior, while boys tend to be physically aggressive towards others. At the same time, significant correlations were revealed between the temperament characteristics and aggressiveness.

Despite the interest in obtained results and their significance, the study concerned only real space, and gender differences included only the characteristics of the biological sex.

Representatives of the psychosocial approach in the course of the study have found a stable correlation between gender and self-assessment of various characteristics of aggressiveness of adolescent girls and boys (Kasimova 2015; Kirienko 2014; Knyshev 2009; Stepanyan et al. 2007).

G.L. Knyshev (2009) described such features of students' communication as aggressiveness and sociability, as well as the dynamics of their changes. The scientist found a negative correlation between the level of physical aggression and verbal aggression with the value of sociometric status. The largest number of rejected adolescents with pronounced manifestations of emotionally reactive aggressiveness occurred in the sociometric structure of the studied groups.

It should be noted that these approaches do not fully describe the mechanisms of teenage aggression in real space and most often do not work in virtual space. Within the framework of these approaches, it is difficult to analyze the aggressive behavior of adolescents such as trolling, bullying, and flame. Taking into account the symbolism and simulative nature of the Internet space, let us consider the nature of aggressive behavior of adolescents on social networks.

A.I. Erzin (2013) considers aggression as a form of proactive behavior, A.I. Luchinkina (2018) analyzes the tendency of users to trolling.

A.I. Luchinkina (2019) notes in her studies of Internet socialization the symbolism of self-presentation of adolescents prone to trolling or bullying on social networks. The researcher draws attention to the aggressiveness of images and metaphors that they contain.

According to the characteristics of communicative behavior, I.S. Luchinkina (2018) considers the aggressiveness of adolescents on social networks as one of the mechanisms of 
psychological defense. I.S. Luchinkina associates aggressiveness with cognitive bias of thinking.

In the works of other authors, aggressiveness of adolescents is associated with high hostility (Semenyuk 2003).

We consider these results to be important for our study and assume that the aggressiveness of adolescents has gender differences and is revealed within chosen strategies of psychological defense, the formed communicative attitudes, the level of hostility, the type of self-presentation of an individual on social networks through antisocial activities, including trolling.

\section{Materials and Methods}

The study has been carried out for over 2 years (November 2018 - November 2020). The study involved 268 adolescents aged 13-14 years. All participants were students of Crimean schools and active users of social networks. 132 of them were males and 136 were females.

The study was carried out in two stages. At the first stage, using A. Assinger's methodology "Assessment of Aggressiveness in Relationships", respondents with a high level of aggressiveness were identified. Then we formed gender subgroups using Bem SexRole Inventory. The method was carried out twice for real and virtual space.

At the second stage of the study we studied self-presentation of representatives of each of the identified subgroups on social networks (content and intent analysis of the page content on social networks), the level of hostility (Cook-Medley methodology "Diagnosis of hostility"), inclination to trolling ("Attitude towards Trolling" Luchinkina I.S.), strategies of psychological defense (V.V. Boyko), empathetic tendency (V.V. Boyko).

The study results showed that a high level of aggressiveness was found in 104 adolescents among 268 respondents. These respondents were included in the experimental group.

\section{Results}

Analysis of gender preferences in the selected group made it possible to divide adolescents into three subgroups according to gender-role types: masculine (27\%), androgynous $(50 \%)$, and feminine $(23 \%)$. It should be noted that among the masculine adolescents there were 14 boys and 10 girls; among the androgynous respondents, 30 were boys and 24 were girls; among feminine respondents there were 8 boys and 18 girls. Thus, the most represented was the group of androgynous adolescents.

In the virtual space the number of teenagers experimenting with their gender is increasing. Thus, the number of feminine respondents is increasing both among girls (20 respondents) and among boys (10 respondents). At the same time, the number of androgynous adolescents is decreasing (boys - 26, girls - 19). The number of masculine girls (14 respondents) and boys (15 respondents) is increasing.

At the second stage, certain characteristics in the virtual space were studied in all groups of adolescents.

A comparative analysis of the empathy of adolescents with different types of gender identity in virtual space is presented in Figure 1. 


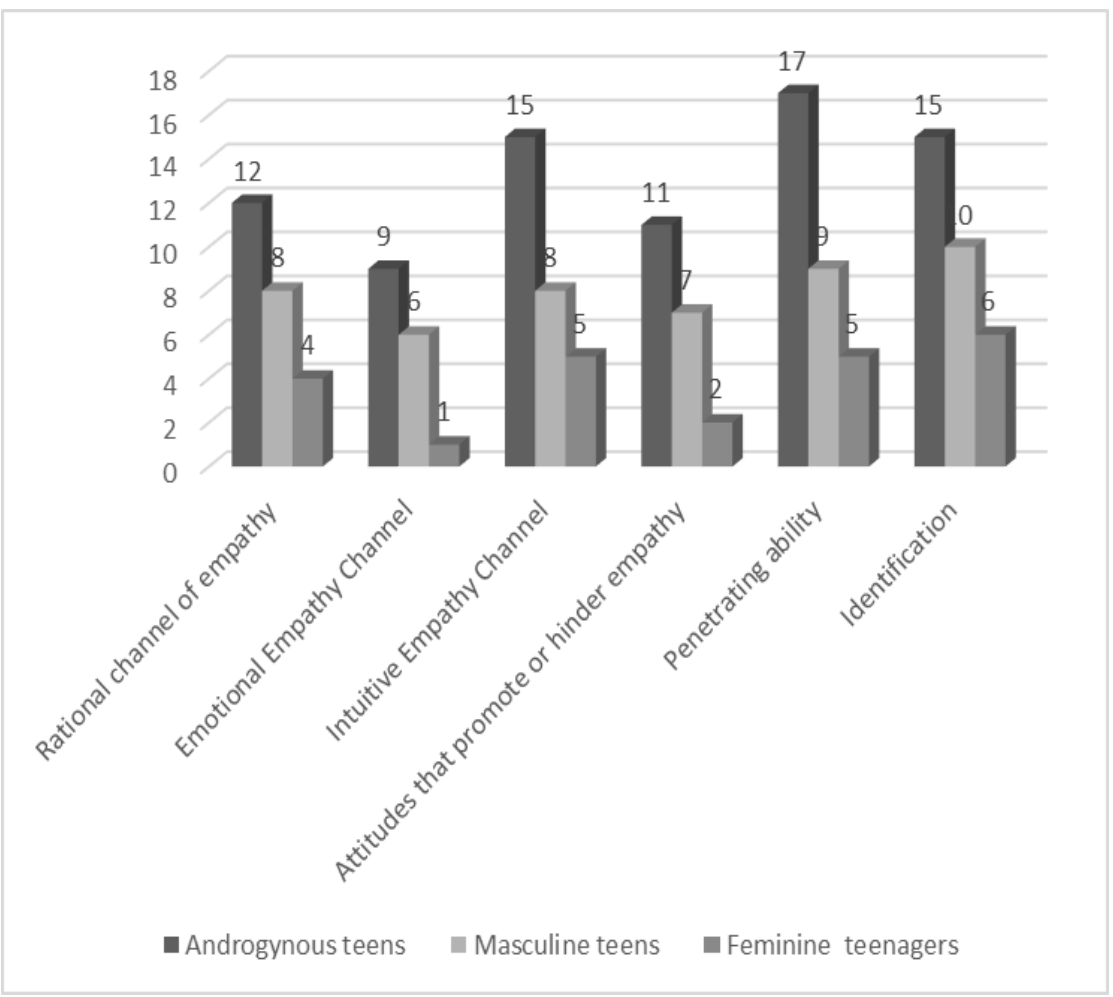

Fig. 1. Analysis of the empathy of adolescents with different types of gender identity in virtual space.

At the second stage, the cognitive, motivational, and affective components of a person's communicative behavior in the Internet space were empirically investigated. At this stage we used following methods: the "Personality in Virtual Space" methodology (A. I. Luchinkina), the "Identification of Awareness of the Various Components of a Motive" methodology (E.P. Ilyin) to study the motivation for using the network. We used author's research questionnaire "Cognitive distortion during online communication" to study dominant cognitive bias of personality; diagnostic methodology of a communicative attitude (V.V. Boyko) to identify the characteristics of the cognitive component of communicative behavior in the Internet space. We also used a reflexivity diagnostic method (A.V. Karpov) to identify the characteristics of the emotional-volitional sphere of communicants, content analysis; $G$. Eysenck's "Self-assessment of mental states" methodology.

As one can see in Figure 1, there are significant differences at $p=0.05$ in the types of empathy among adolescents of different gender groups in the virtual space. Androgynous adolescents are the most empathic; they empathize less and easily perceive aggression towards others without seeking to protect. Muscular adolescents showed an average level of empathy while adolescents with a feminine type have the lowest values for all types of empathy, which can lead to increased conflict in communication.

The results of the study of avatars on users' pages on the social network "VKontakte" are shown in Table 1.

Table 1. Results of the study of avatars and statuses.

\begin{tabular}{|l|c|c|c|}
\hline \multicolumn{1}{|c|}{ Avatar type, \% / gender } & Masculine & Androgynous & Feminine \\
\hline Is absent & 14 & 12 & 0 \\
\hline Photo & 21 & 18 & 21 \\
\hline Animals & 24 & 19 & 5 \\
\hline
\end{tabular}




\begin{tabular}{|l|c|c|c|}
\hline $\begin{array}{l}\text { Heroes of films and fantasy, } \\
\text { superheroes }\end{array}$ & 2 & 8 & 12 \\
\hline Celebrities, politicians & 2 & 2 & 10 \\
\hline Material objects & 19 & 16 & 10 \\
\hline Shocking & 2 & 8 & 26 \\
\hline Humorous & 15 & 8 & 5 \\
\hline Nature & 0 & 1 & 3 \\
\hline Part of the body & 1 & 8 & 8 \\
\hline
\end{tabular}

The study showed significant differences at $\mathrm{p}<0.01$ in the choice of avatars between aggressive adolescents of different genders. Most feminine adolescents choose photos of themselves or shocking, angry images as avatars. Androgynous teenagers are characterized by avatars depicting animals, photos of themselves, material objects. The most popular avatars among masculine adolescents are material objects (cars, motorcycles, etc.), as well as funny images.

There are significant differences between the subgroups according to the chosen characteristics. Thus, the aggressive behavior of masculine adolescents is characterized by the highest indicators of aggressiveness. The aggressive behavior of feminine adolescents is characterized by high rates of hostility and cynicism. Moderate aggressiveness is a characteristic of androgynous adolescents. The significant differences can be noticed between the masculine and feminine subgroups of adolescents.

The study of preferences in trolling among the respondents of the subgroups allowed us to make some conclusions about the various types of trolling. Therefore, masculine teens tend to troll for entertainment. Typically, these adolescents choose their prey being guided by the hunter's instinct.

Androgynous teens are prone to political trolling or interest trolling. Most often, they mock classmates for their hobbies, devalue them.

Feminine teens are prone to physical trolling and gender trolling. They often write offensive comments in these directions.

The study of the dominant strategy of psychological defense in communication among adolescents from the experimental group revealed significant differences at $p<0.01$.

Table 2. Gender distributions of dominant strategies of psychological defense, $\%$.

\begin{tabular}{|l|c|c|c|}
\hline $\begin{array}{c}\text { Dominant psychological } \\
\text { defense strategy }\end{array}$ & Masculine & Androgynous & Feminine \\
\hline Peacefulness & 58 & 31 & 0,00 \\
\hline Avoidance & 28 & 54 & 17 \\
\hline Aggression & 14 & 15 & 83 \\
\hline
\end{tabular}

As can be seen from the results shown in Table 2, the most relevant strategy of psychological defense for masculine adolescents is peacefulness. Despite the aggressiveness of this subgroup, these teens are less prone to trolling and bullying on social networks than feminine ones. At the same time, the dominant strategy of psychological defense for feminine adolescents is aggression, which can be revealed in categorical judgments, uncompromising, offensive content. The most important strategy for androgynous adolescents is avoidance.

\section{Discussion}

The most relevant questions for discussion were the following:

1. What is typical for aggressive behavior of adolescents in social networks?

The answer to this question undoubtedly lies in the field of methodology and reflects the general concept of studying the communicative behavior of adolescents in a virtual environment. Aggressiveness in adolescents is defined as a personality trait that develops in 
society. Analysis of scientific literature and our preliminary studies have shown that the aggressiveness of adolescents has gender differences and is implemented according to the chosen strategies of psychological defense, the level of hostility and self-presentation of the individual in social networks through antisocial activities, including trolling. These components characterize adolescent aggressiveness in social networks. Moreover, due to anonymity in the virtual space, ethical norms and rules of interaction adopted in real society are often violated. Lack of responsibility for their actions leads to insults and cruelty.

2. What are the gender characteristics of adolescent aggression in social networks connected with?

Researchers of adolescent aggression in real space have repeatedly noted that boys are more prone to physical aggression, while girls are more prone to verbal aggression. However, the incorporeality of the virtual space creates equal conditions for all biological sexes in establishing communications. Therefore, it is important to analyze the features of communication not from the point of view of biological sex, but from the point of view of gender. Experiments with sex, typical of adolescents and widespread in the virtual space, lead to the emergence of new forms of behavior and outbursts of aggression in some cases due to lack of experience in responding to non-standard situations. The study showed that the most aggressive are feminine adolescents.

\section{Conclusions}

The results of the study led to the following conclusions:

1. Aggressive behavior of masculine adolescents is characterized by the highest rates of propensity to physical aggression. Trolling as an entertainment is typical for adolescents in this group. The dominant strategy of psychological defense is peacefulness. The statuses are "Leading social activities, work, and study".

2. Aggressive behavior of feminine adolescents is characterized by high rates of hostility and cynicism, gender trolling, family trolling, trolling based on opponent's appearance, high rates of aggression as psychological defense strategy, low level of empathy.

3. Androgynous adolescents are characterized by moderate aggressiveness, trolling based on opponent's appearance, the psychological defense strategy is avoidance.

\section{References}

1. A.I. Erzin, Psychologist 3, 326-337 (2013) doi: 10.7256/2306-0425.2013.3.640

2. E.P. Ilyin, Psychology of aggressive behavior (Piter, St. Petersburg, 2014)

3. I.S. Isaeva, Individual-typological features of sociability and aggressiveness of cadets of a military university (RUDN, Moscow, 2008)

4. T.V. Kalinina, N.S. Urivskaya, Actual Prob. of Mod. Ped. 1, 8-11 (2017)

5. Z.G. Kasimova, Concept 10, 111-115 (2015)

6. A.A. Kirienko, Individual and personal characteristics of the aggressiveness of adolescents of various types of personality orientation (RUDN, Moscow, 2014)

7. G.L. Knyshev, Collection of scientific articles dedicated to the 70th anniversary of A. I. Krupnov, 269-277 (2009)

8. P.A. Kovalev, Age-sex characteristics of the reflection in the consciousness of the structure of one's own aggressiveness and aggressive behavior (Abevega, St. Petersburg, 1996) 
9. A.B. Kryuchkova, Specificity of the correlation of aggressiveness with the properties of temperament in boys and girls (RUDN, Moscow, 2010)

10. A.I. Luchinkina, I.S. Luchinkina, Russian psychological journal 1(16), 128-143 (2019) doi: 10.21702/rpj.2019.1.6

11. A.I. Luchinkina, Pedagogy and Psychology of Education 4(2), 68-76 (2018) doi: 10.18413/2313-8971-2018-4-2-0-7

12. I.S. Luchinkina, Humanitarian sciences 4(44), 153-158 (2018)

13. T.V. Nechepurenko, Gender differences in the manifestations of students' aggressiveness (RUDN, Moscow, 2009)

14. L.M. Semenyuk, Psychological features of aggressive behavior in adolescents and conditions for its correction (Flinta, Moscow, 2003)

15. A.Yu. Stepanyan, L.S. Stepanyan, V.G. Grigoryan, Ananyev readings 1, 616-618 (2007)

16. N.Yu. Zharnovetskaya, Age-sex characteristics of perception of aggressiveness (Print house RPGU named after A.I. Herzen, St. Petersburg, 2006)

17. I.V. Zhuikova, Univ. Schol. Notes 12(118), 257-261 (2014) doi: 10.5930/issn.19944683.2014.12.118.p257-261 